\title{
Effect of Allotriploidization on Development of the Hybrids between Female Chum Salmon and Male Brook Trout*1
}

\author{
Katsutoshi Arai*2 \\ (Accepted October 9, 1985)
}

\begin{abstract}
Mature ova of chum salmon Oncorhynchus keta were inseminated with milt from brook trout Salvelinus fontinalis and a portion of the eggs were treated with hydrostatic pressure in order to block the second polar body extrusion and to induce allotriploidy. The embryos from eggs exposed to the hydrostatic pressure shock were more viable than embryos from the usual hybridization. Karyological studies revealed that the latter inviable hybrids had exactly the same intermediate diploid karyotype as that of between two parental species, while the former viable hybrids had the allotriploidy comprising two sets of the maternal haploid complement and one set of the paternal haploid complement. A small number of viable larvae which occurred in the usual hybridization were found to be spontaneous allotriploids in biochemical studies using isozymes as gene markers. These facts proved that allotriploidization can lead to increased survival of developing embryos in some hybridization between salmonid species.
\end{abstract}

It has been recognized in a few reports on the artificial induction of triploid hybrids in salmonids that triploid interspecific hybrids were more viable than diploid hybrids. ${ }^{1,2}$ Spontaneous triploids have been observed in interspecific hybrids between rainbow trout Salmo gairdneri and brook trout Salvelinus fontinalis. ${ }^{3,4}$ Thorgaard $^{5)}$ suggested that spontaneous triploids might be related to better survival of triploid hybrids than diploid hybrids and triploidy might lead to increased viability in interspecific hybrids. A similar observation and conclusion were obtained in a previous work. ${ }^{6)}$ In allotriploids between female chum salmon Oncorhynchus keta and male Japanese char Salvelinus leucomaenis, having maternal diploid $(2 n=74)$ and paternal haploid $(n=42)$ complements, a drastic recovery of the developmental potential was found in contrast with the abnormal embryogenesis of the diploid hybrid which had the theoretical intermediate diploid complement $(2 n=79)$ between the two species. ${ }^{3}$ The artificial triploidization, however, was not effective in increasing survival rate of the inviable hybrids Japanese char $O x$ chum salmon $\delta$ which consisted of aneuploid cells with hypodiploid chromosome numbers. ${ }^{(3)}$ The embryos thus induced to triploidization showed abnormal chromosome numbers smaller than the expected allotriploidy. ${ }^{8}$ These facts suggest that the viability-increasing effect of allotriploidization may be related to the cytogenetic characteristics of interspecific hybrids. The hybridization between chum salmon and brook trout was found to be inviable in previous research $^{(2)}$ and may be useful as a model. In the present study, artificial allotriploidization was attempted in hybrids between female chum salmon and male brook trout by preventing the extrusion of the second polar body with hydrostatic pressure shock. The developmental potentials, chromosome numbers, karyotypes and isozyme phenotypes were examined to evaluate success and effect of the allotriploidization.

\section{Materials and Methods}

Chum salmon Oncorhynchus keta were captured in the Katagishi river, Kamaishi, Iwate Prefecture at November, 1983. Mature ova were collected from two female chum salmon, then mixed and again divided into two batches. One batch was inseminated with milt from a brook trout Salvelinus fontinalis. This brook trout had been reared in the pond of the Tadami Fish Culture Station of Kitasato University, Tadami, Fukushima Prefecture and transported to the laboratory of Kitasato University, Sanriku, Twate Prefecture, for the artificial hybridization.

Eggs from both the chum salmon $9 \times$ chum

*1 Supported in part by grant-in-aids from the Japan Ministry of Education, Science, and Culture.

*2 Kitasato University, School of Fisheries Sciences, Sanriku, Iwate 022-01, Japan (堷挑克俊: 北里大学水 磨学部). 
Table 1. Survival rates (\%) of embryos of control and HP shocked groups in the chum salmon and chum salmon $q$ ㅇ $\times$ brook trout $\hat{\sigma}$ hybridization

\begin{tabular}{|c|c|c|c|c|c|c|c|c|c|c|c|c|c|c|}
\hline \multicolumn{2}{|c|}{ Cross } & \multirow{2}{*}{ Group } & \multirow{2}{*}{$\begin{array}{c}\text { No. of } \\
\text { embryos } \\
\text { examined }\end{array}$} & \multicolumn{10}{|c|}{ Days after insemination } & \multirow{2}{*}{$\begin{array}{l}\text { Date of } \\
\text { hatching }\end{array}$} \\
\hline q & $\overrightarrow{0}$ & & & 10 & 20 & 30 & 40 & 50 & 60 & 70 & 80 & 90 & 100 & \\
\hline \multirow{2}{*}{\multicolumn{2}{|c|}{ chum $\times$ chum }} & Control & 255 & 99.2 & 98.8 & 94.5 & 86.7 & 78.8 & 75.7 & 74.1 & 72.5 & 67.5 & 63.1 & 93 \\
\hline & & HP shocked & 132 & 99.2 & 99.2 & 96.2 & 90.2 & 79.5 & 77.3 & 71.2 & 70.0 & 68.0 & 62.0 & 93 \\
\hline \multirow{2}{*}{\multicolumn{2}{|c|}{ chum $\times$ brook }} & Control & 148 & 100.0 & 95.3 & 84.6 & 67.6 & 48.6 & 39.2 & 37.2 & 29.7 & 21.6 & 6.1 & 76 \\
\hline & & HP shocked & 132 & 1000 & 100.0 & 91.7 & 86.4 & 82.6 & 82.6 & 79.5 & 75.8 & 72.0 & 58.3 & 76 \\
\hline
\end{tabular}
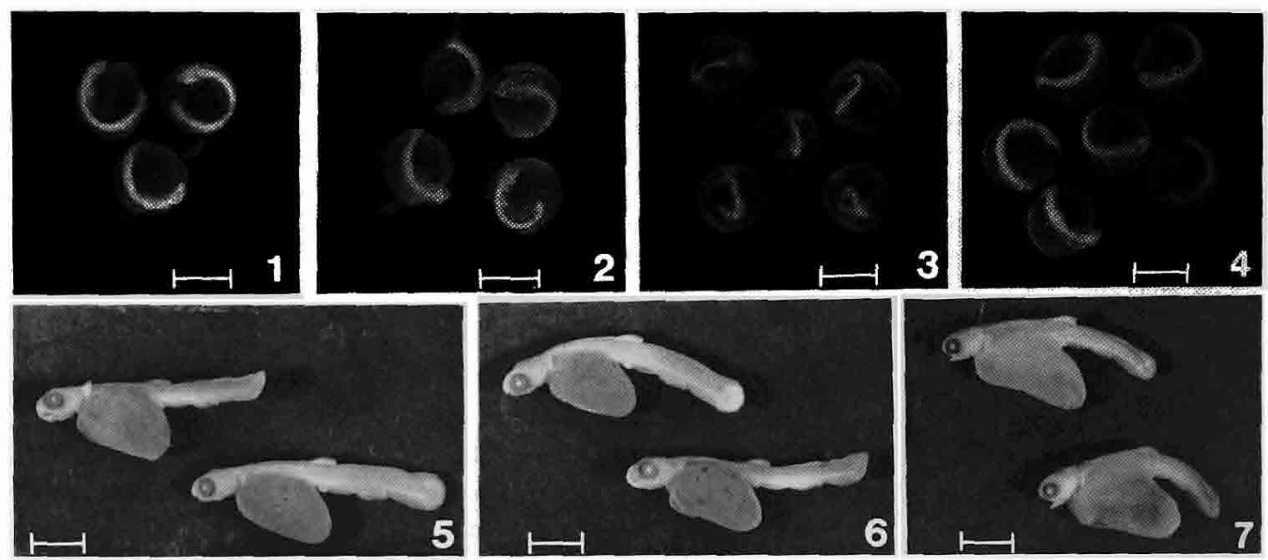

Figs. 1-7. External appearances of embryos and larvae from control and HP shocked group of chum salmon and chum salmon $q \times$ brook trout $\hat{\delta}$ hybridization. Each scale indicates $5 \mathrm{~mm}$.

Fig. 1. Eyed embryos developed from control eggs of chum salmon.

Fig. 2. Eyed embryos developed from HP shocked eggs of chum salmon.

Fig. 3. Abnormal embryos developed from control eggs of chum salmon $q \times$ brook trout $\delta$ hybridization. Note microcephalia, microphthalmia and dwarf embryonic body.

Fig. 4. Eyed embryos developed from HP shocked eggs of chum salmon $q \times$ brook trout ${ }^{\star}$ hybridization.

Fig. 5. Hatched larvae developed from control eggs of chum salmon.

Fig. 6. Hatched larvae developed from HP shocked eggs of chum salmon.

Fig. 7. Hatched larvae developed from HP shocked eggs of chum salmon $q \times$ brook trout $\hat{\sigma}$ hybridization.

salmon $\delta^{t}$ and the chum salmon $q \times$ brook trout $\delta^{t}$ were further divided into two groups. Eggs of one group were treated with hydrostatic pressure (HP) shock in order to induce triploidy according to the same method as described in a previous work. ${ }^{8)}$ Fifteen min after insemination, eggs were exposed for seven min to $650 \mathrm{~kg} / \mathrm{cm}^{2}$ of hydrostatic pressure. The other group of eggs was used as the intact control without HP shock.

Each group was subdivided into two groups. For the morphological, chromosomal and electrophoretic observations, specimens were taken from one subdivided group, while the other was checked for viability by monitoring the daily survival rate. Embryos were incubated in normal running freshwater. The water temperature was $5.3^{\circ} \mathrm{C}$ in average but fluctuated between 3.0 and $8.7^{\circ} \mathrm{C}$ in the daily measurement taken each noon.

Five to ten embryos in each group were taken and fixed with Bouin's fixative at around the stages of eye-pigmentation and hatching. Chromosome preparation was made on the 21 to 24 days old embryos according to the procedure described in a previous paper. ${ }^{8)}$ Starch gel electrophoresis of phosphoglucomutase (PGM) (EC 2.7.5.1) and 6phosphogluconate dehydrogenase (6PGDH) (EC 1.1.1.44) was performed on the muscle tissues of the parent fish and hatched larvae. Electrophoresis and histochemical procedures were the same as those used in a previous study. ${ }^{6)}$ Nomenclature of isozymes, alleles and genetic loci was also the same as that used in the previous work. ${ }^{8}$ ) 


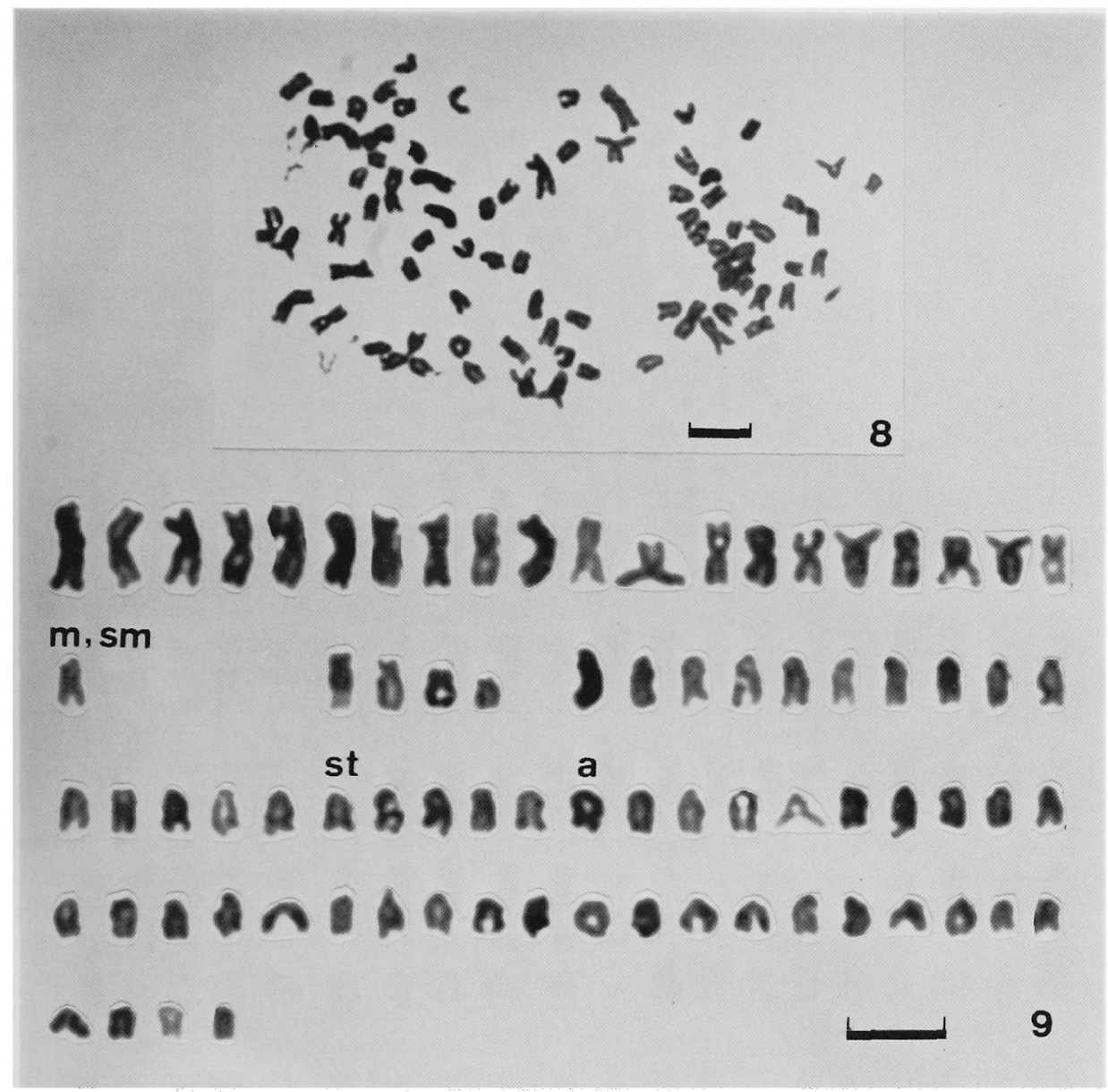

Figs. 8, 9. Chromosome complement of diploid hybrid chum salmon $q \times$ brook trout $\delta$. Each scale indicates $10 \mu \mathrm{m}$.

Fig. 8. Metaphase spread with 79 chromosomes in the embryo from control group of chum salmon $q \times$ brook trout $\delta$ hybridization.

Fig. 9. Karyogram of diploid hybrid chum salmon $q \times$ brook trout $\delta$. The karyotype consists of 21 meta- or submetacentric (m, sm), 4 subtelocentric (st) and 54 acrocentric elements (a).

\section{Results}

\section{Developmental Studies}

Survival rates and dates of hatching during the development of control and HP shocked embryos in the parental and the hybrid crosses are given in Table 1. No significant difference was observed in the survival rates between the control and the HP-group of the chum salmon. Both groups began to hatch at 93 days after insemination at the present incubating condition.

In the hybrid embryos between female chum salmon and male brook trout, the survival rates of the HP-group were quite higher than those of the untreated control group (Table 1). Embryos of the HP-group started to hatch at 76 days after insemination. In contrast, only nine embryos in the untreated hybrid group hatched in the same period, while the other embryos in the same group died up to 100 days after insemination (Table 1). Hatched larvae in both groups survived and continued to grow up to three months after hatching.

As shown in Figs. 1 and 2, no difference was recognized in the morphology at the eyed stage between the control and the HP-group in the chum salmon. In contrast, there was a conspicuous difference in the appearance of embryos between the control and the HP-group in the hybrids of chum salmon $q \times$ brook trout $\delta$. The embryos 


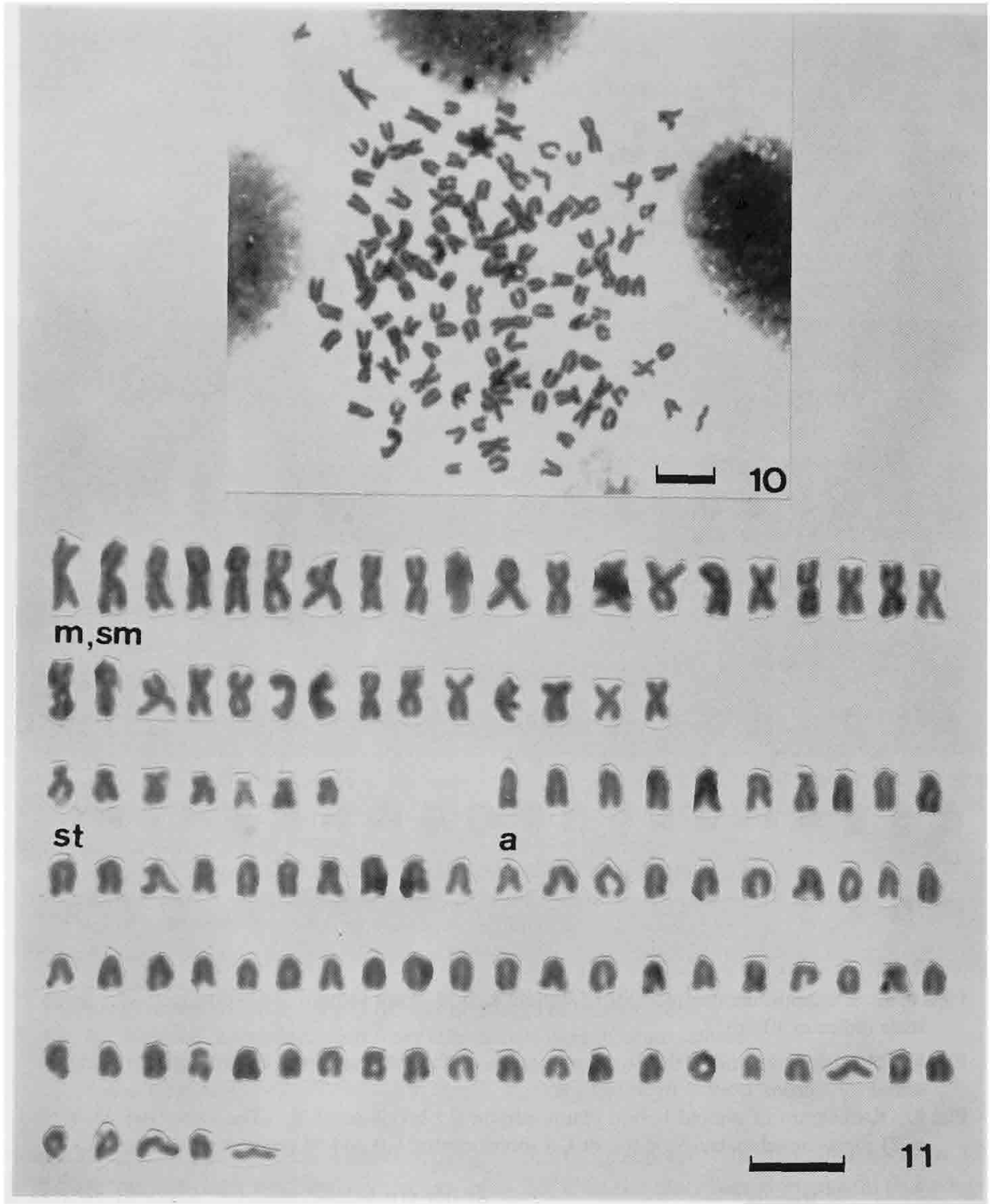

Figs. 10, 11. Chromosome complement of allotriploid hybrid chum salmon $\uparrow \times$ brook trout $\hat{\delta}$. Each scale indicates $10 \mu \mathrm{m}$.

Fig. 10. Metaphase spread with 116 chromosomes in the embryo from HP-group of chum salmon q $\times$ brook trout $\delta$ hybridization.

Fig. 11. Karyogram of allotriploid hybrid chum salmon $q \times$ brook trout $\hat{\sigma}$. The karyotype consists of 34 meta- or submetacentric $(\mathrm{m}, \mathrm{sm}), 7$ subtelocentric (st) and 75 acrocentric elements (a).

from the untreated control group had stunted bodies with microcephalia and microphthalmia (Fig. 3), while the HP-group appeared to have the normal morphology at the same stage (Fig. 4). Hatched larvae were obtained in the control (Fig. 5) and the HP-group (Fig. 6) of the chum salmon, and there was no difference in their external appearances and sizes. Quite a few larvae in the untreated hybrid group hatched. They had the same normal appearance as observed in the HPgroup (Fig. 7). 

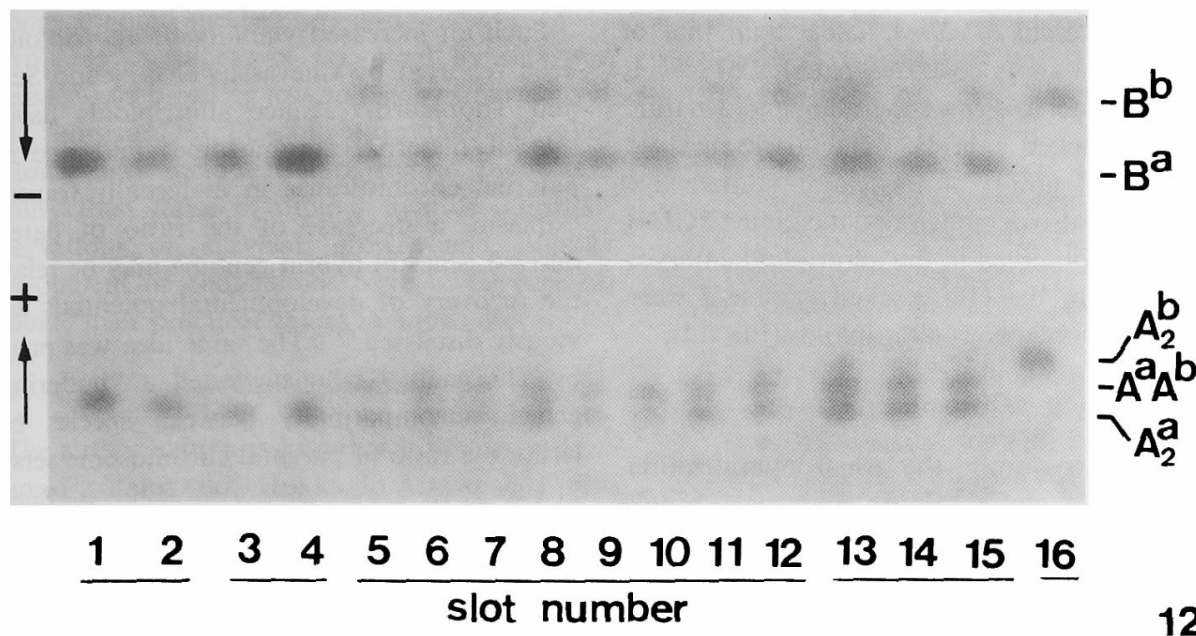

Fig. 12. Zymograms of phosphoglucomutase (PGM) (upper) and 6-phosphogluconate dehydrogenase (6PGDH) (lower) isozymes in muscle tissues of parent fish and hatched larvae. 1, 2, larvae from control group of chum salmon; 3, 4, larvae from HP-group of chum salmon; 5-12, larvae from HP-group of chum salmon $q \times$ brook trout $\delta$ hybridization; 13-15, Survived larvae from control group of chum salmon $q \times$ brook trout $\hat{\delta}$ hybridization; 16 , male brook trout as paternal fish.

\section{Chromosomal Studies}

The chromosomal observations on six embryos in the HP-group of the chum salmon revealed the triploidy $(3 n=111)$ in all embryos examined, while those on three embryos in the control proved normal diploidy $(2 n=74)$. Chromosome counts on a total of eleven embryos from the hybridization between female chum salmon and male brook trout showed that ten embryos had a diploid complement comprised of 79 chromosomes (Fig. 8), while the other had 116 chromosomes. The karyotype of an embryo with 79 chromosomes consisted of 21 meta- or submetacentric, 4 subtelocentric and 54 acrocentric chromosomes (Fig. 9). Chromosome counts on a total of twelve embryos from the HP-group of the chum salmon o $\times$ brook trout $\delta$ revealed that eleven embryos had the triploid chromosome number $(3 n=116)$ (Fig. 10), while the other was the diploid hybrid $(2 n=79)$. The karyotype of an embryo with 116 chromosomes consisted of 34 meta- or submetacentric, 7 subtelocentric and 75 acrocentric elements (Fig. 11).

\section{Biochemical Studies}

The isozyme phenotypes of phosphoglucomutase (PGM) were examined in hatched larvae. As shown in Fig. 12, maternal chum salmon and paternal brook trout had mutually distinguishable $\mathrm{PGM}^{\mathrm{B}} \mathrm{B}^{\mathrm{n}}$ and $-\mathrm{B}^{\mathrm{b}}$ isozymes, indicating an allelic difference at $P g m-B$ locus between the two species. In the chum salmon, there was no electrophoretic difference in the PGM phenotype between the control and the HP shocked larvae (Fig. 12). Although the hybrid embryos developed from eggs subjected to the HP shock had two different PGM isozymes as shown in Fig. 12, the maternally derived PGM-B ${ }^{a}$ had twice the staining intensity than the paternally derived PGM- ${ }^{b}$. These PGM phenotypes indicate that the hatched larvae had two alleles of maternal $P g m-B_{a}$ and one allele of paternal $P g m-B_{b}$ at $P g m-B$ locus in their genomes. The allotriploid nature was also observed in the hatched larvae developed from the untreated chum salmon $q \times$ brook trout $\hat{\sigma}$ group (Fig. 12).

There was an electrophoretic difference in mobilities of gene products by the $6 \mathrm{Pgdh}-\mathrm{A}$ locus between the chum salmon and the brook trout (Fig. 12). No distinction was observed in 6PGDH phenotype between the control and the HP shocked chum salmon (Fig. 12). The hybrids from the HP-group had the isozyme pattern consisting of $6 \mathrm{PGDH}-\mathrm{A}_{2}^{\mathrm{a}}$ homodimer, $-\mathrm{A}^{\mathrm{a}} \mathrm{A}^{\mathrm{b}}$ heterodimer and $-\mathrm{A}_{2}^{b}$ homodimer (Fig. 12). The staining intensities of these three isozymes were distributed according to a ratio of almost $4: 4: 1$ (Fig. 12). These phenotypes proved twice the presence of $6 \mathrm{PGDH}-\mathrm{A}^{\mathrm{a}}$ subunit polypeptides 
coded by maternal $6 P g d h-A_{a}$ allele than that of 6PGDH-A ${ }^{\mathrm{b}}$ subunits coded by paternal $6 P g d h-A_{b}$ allele. This fact indicates the allotriploid nature of the HP shocked hybrids. The larvae survived in the control group of the chum salmon $q \times$ brook trout also demonstrated the same $6 \mathrm{PGDH}$ phenotype as observed in the allotriploid embryos developed from the HP shocked eggs and were found to be spontaneous allotriploids (Fig. 12).

\section{Discussion}

Most embryos from the usual hybridization between female chum salmon and male brook trout were found to be inviable except for a small number of laryae with normal appearances. Almost identical survival rates of this hybridization were observed in the previous study. ${ }^{6)}$ It was reported that the karyotype of chum salmon consisted of 26 meta- or submetacentric $(\mathrm{m}, \mathrm{sm}), 6$ subtelocentric (st) and 42 acrocentric chromosomes (a) and that of brook trout consisted of 16 meta- or submetacentric, 2 subtelocentric and 66 acrocentric chromosomes. ${ }^{0)}$ It is evident from the present chromosomal study that the inviable hybrids between female chum salmon and male brook trout had the exact same intermediate karyotype $(2 n=79,21 \mathrm{~m}, \mathrm{sm}+4 \mathrm{st}+54 \mathrm{a})$ being equal to an exact sum of each haploid complement of the parental two species. It is interesting that the karyotype of the chum salmon $q \times$ brook trout $f$ clearly differed from that of the reciprocal brook trout $9 \times$ chum salmon $\hat{o}$ which was reported to comprise aneuploid cells with various hypodiploid numbers. ${ }^{6)}$

The chromosomal study revealed that the hydrostatic pressure (HP) shock successfully induced triploidy in the chum salmon. In the hybridization between female chum salmon and male brook trout, most embryos developed from eggs exposed to the same HP shock had the allotriploid karyotype $(3 n=116,34 m, s m+7 s t+75 a)$ comprised of the diploid complement of chum salmon $(2 \mathrm{n}=74,26 \mathrm{~m}, \mathrm{sm}+6 \mathrm{st}+42 \mathrm{a})^{2)}$ and the haploid complement of brook trout $(n=42,8 \mathrm{~m}$, $s m+1 s t+33 a) .^{8)}$ The isozymic study on hatched larvae of the same group also proved their allotriploid nature. Therefore, most embryos from the HP-group in the hybrid were allotriploidy. The fact most worth noting was that these allotriploid hybrids were normal in appearance and viable when compared with the diploid hybrids which showed lethal malformations during embryogenesis.
Such an increased viability of allotriploids has been reported by Chevassus et al ${ }^{1)}$ and Scheerer and Thorgaard. ${ }^{2)}$ Since allotriploids have two sets of maternal chromosomes and one set of paternal chromosomes in maternally transmitted ooplasm, a decrease of the ratio of paternally derived genes in hybrid genome may be related to the recovery of developmental potentials as previously discussed. ${ }^{8)}$ The same idea was proposed by Thorgaard" who suggested a "buffering" of genetic incompatibility between species by altering the ratio of parental chromosome sets from $1: 1$ to $2: 1$.

However, the function of maternal ooplasm must be considered because the hybrid incompatibility may not only act between two parental genomes but also between paternal genome and maternal cytoplasm. In the previous work, the author suggested the possibility that nucleocytoplasmic incompatibility might eliminate paternally derived chromosomes during the development of Japanese char $q \times$ chum salmon $\hat{\delta}$ and brook trout $\phi \times$ chum salmon $\delta$. Therefore, success of the production of viable hybrids by triploidization may depend on the degree of nucleo-cytoplasmic incompatibility between the two parental species and the cytogenetic character of the resultant hybrids. At present, no further discussion is possible on the mechanisms responsible for the viability-increasing effect of allotriploidy. Additional experiments, especially the study of various kinds of allotriploids with various kinds of genomic and nucleo-cytoplasmic ratio between species would be necessary to explain and generalize the mechanisms.

Regarding hybridization of fish, Chevassus ${ }^{10)}$ pointed out that it seemed very hazardous to call every individuals from hybridization a "hybrid", the risk of error being higher the lower the survival rate. The present study also revealed that a small number of hybrid larvae between female chum salmon and male brook trout which survived were spontaneous allotriploids. Spontaneous triploids have also been observed in rainbow trout $\times$ brook trout $\delta^{3,4)}$ while spontaneous gynogenesis has been reported in coho salmon Oncorhynchus kisutch $9 \times$ brook trout $\delta^{77,8)}$ and brook trout $q \times$ brown trout Salmo trutta $\delta^{\prime} .^{\text {) }}$ It is worth noting that the hybridization using brook trout may tend to induce unusual developments such as spontaneous allotriploid and hybrid gynogenesis. These facts suggest a need to re-examine the results reported in former hybridization studies 
among salmonid species.

Probable usefulness of polyploid hybrids was discussed as a breeding technology by Chevassus ${ }^{10}$ and Thorgaard.") Possible advantages of allotriploids may include higher hybrid vigor and sterility than those of diploid hybrids. Further observations on survival, growth and gonadal development of allotriploids have to be done to evaluate their practical values in aquaculture.

\section{Acknowledgements}

The author expresses his sincere thanks to Professor F. Yamazaki, Hokkaido University, who kindly read the manuscript and gave valuable criticism with important advices. The author is also very grateful to Professor K. Fujino and Professor Hitoshi Ida, Kitasato University, for their kind help with the collection of parental salmonid fishes. A debt of gratitude is also owed to Dr. H. Onozato, National Research Institute of Aquaculture, for his valuable suggestions on the present study. Thanks are also extended to Miss $Y$.
Kimura and Mr. K. Nakano, Kitasato University, for their assistance in the present study.

\section{References}

1) B. Chevassus, R. Guyomard, D. Chourrout, and E. Quillet: Génet. Sel. Evol, 15, 519-532 (1983).

2) P. D. Scheerer and G. H. Thorgaard: Can. J. Fish. Aquat. Sci, 40, 2040-2044 (1983).

3) E. Capanna, S. Cataudella, and R. Volpe: Boll. Pesca, Piscic. Idrobiol, 29, 101-106 (1974).

4) T. Ueda, Y. Ojima, R. Sato, and Y. Fukuda: Bull. Japan. Soc. Sci. Fish, 50, 1331-1336 (1984).

5) G. H. Thorgaard: in "Fish Physiology" (ed. by W. S. Hoar, D. J. Randall, and E. M. Donaldson), Vol. 9, Part B, Academic Press, New York, 1983, pp. $405-434$.

6) K. Arai: Mem. Fac. Fish. Hokkaido Univ., 31, 1-94 (1984).

7) T. Uyeno: Japan.J. Ichthyol., 19, 166-171 (1972).

8) H. Onozato: Fish Genet. Breed. Sci., 6, 11-18 (1981) (in Japanese).

9) K. Buss and J. E. Wright: Prog. Fish Cult, 18, 149-158 (1956).

10) B. Chevassus: Aquaculture, 33, 245-262 (1983). 